\title{
EFECTOS A CORTO PLAZO DE LA CONTAMINACIÓN ATMOSFÉRICA SOBRE LA MORTALIDAD. RESULTADOS DEL PROYECTO EMECAM EN ZARAGOZA, 1991-1995
}

Federico Arribas Monzón (1), Tomás Alcalá Nalvaiz (2), José M.a Abad Díez (3), Mercedes Navarro Elipe (4), María del Carmen Martos Jiménez (1), M. ${ }^{a}$ José Rabanaque Hernández (2), Sara Zapatero Molinera (1) y Enrique Muniesa Casamayor (1)

(1) Diputación General de Aragón.

(2) Universidad de Zaragoza.

(3) Hospital Clínico Universitario de Zaragoza.

(4) Ayuntamiento de Zaragoza.

\section{RESUMEN}

Fundamento: Evaluar el impacto a corto plazo de la contaminación sobre la mortalidad respiratoria en la ciudad de Zaragoza, en el periodo 1991-95, e identificar si hay diferencias en función de la edad y época del año.

Métodos: La asociación de concentraciones diarias de humos y $\mathrm{SO}_{2}$ con las defunciones diarias por enfermedades respiratorias (CIE-9 460-486) y enfermedad pulmonar obstructiva crónica y afines EPOC-EA (490-496), se analizó aplicando modelos de Poisson, siguiendo el protocolo del proyecto EMECAM. Se investigaron posibles diferencias del efecto en mayores y menores de 70 años y según semestre. Se calcularon riesgos relativos (RR) e intervalos de confianza al 95\% (IC $95 \%$ ) para incrementos de contaminante de $10 \mu \mathrm{gr} / \mathrm{m}^{3}$.

Resultados: Se encontró asociación entre mortalidad respiratoria y humos (RR 1,028 $\mathrm{IC}_{95 \%}$ 1,006-1,051), siendo el riesgo mayor en semestre cálido. En mayores de 70 años, la relación se mantuvo en este semestre y fue negativa en menores de 70 . Los RRs de la mortalidad por EPOC-EA fueron globalmente de $1,038\left(\mathrm{IC}_{95 \%} 1,002-1,075\right)$ y de $1,068\left(\mathrm{IC}_{95 \%}: 1,004-1,137\right)$ para el semestre cálido. La contaminación por $\mathrm{SO}_{2}$ mostró asociación positiva con la mortalidad respiratoria en periodo cálido para todas las edades, RR 1,093 (IC95\%: 1,006-1,187) y en menores de 70 años (RR 1,240 IC $95 \%$ : 1,028-1,496). Los efectos fueron no concluyentes con las neumonías.

Conclusiones: Niveles bajos de contaminación atmosférica pueden producir efectos significativos en la mortalidad respiratoria, especialmente en semestres cálidos y en personas mayores.

Palabras clave: Mortalidad respiratoria. Contaminación atmosférica. Regresión de Poisson.

Correspondencia:

Federico Arribas Monzón

Sección de Vigilancia Epidemiológica

Servicio Provincial de Sanidad de Zaragoza

Ramón y Cajal, 68

50004 ZARAGOZA

FAX: 976- 715076

Correo electrónicn: farribas@aragoh.es

\section{ABSTRACT}

\section{The Short-term Impact of Air Pollution on the Respiratory Mortality. Results of the $E M E C A M$ Project in the city of Saragossa, 1991-1995}

Background: To assess the short-term impact of pollution on the respiratory death rate in the city of Saragossa throughout the 1991-1995 period and to pinpoint whether any differences exists in terms of age and time of the year.

Methods: The relationship of daily concentrations of smog and $\mathrm{SO}_{2}$ to the daily deaths due to respiratory diseases (CIE-9 460-486) and chronic lung blockage disease and similar EPOC-EA (490-496) was analyzed using Poisson models in keeping with the EMECAM procedure. Possible differences in the impact on those below and over age 70 and according to the six-month period in question were researched. Relative risks (RR) and 95\% confidence intervals $\left(\mathrm{CI}_{95 \%}\right)$ WERE CALCULATED FOR $10 \mu \mathrm{gr} / \mathrm{m}^{3}$ rises in pollutant.

Results: A relationship was found to exist between the respiratory and smog death rate (RR $1.028 \mathrm{CI}_{95 \%} 1.006-1051$ ), the highest risk being during the six-months period of warm weather. For those individuals over age 70 , the relationship remained the same throughout this six-month period and was negative for those individuals under age 70. The RR's for the death rate based on EPOC-EA were, overall, 1.038 ( $\mathrm{CI}_{95 \%}$ $1.002-1075)$ and of $1.068\left(\mathrm{Cl}_{95 \%}: 1.004-1.137\right)$ for the six-month period of warm weather. The $\mathrm{SO}_{2}$ pollution showed a positive relationship to the respiratory death rate for the warm period for all ages, RR 1.093 (CI ${ }_{95 \%}$ : 1.006-1.187) and for those under age 70 (RR 1.240 $\mathrm{CI}_{95 \%}: 1.028-1.496$ ). The impact was not conclusive for the cases of pneumonia.

Conclusions: Low levels of air pollution can have a significant impact on the respiratory death rate, especially among the elderly and during the six-month period of warm weather.

Key words: Respiratory. Mortality. Air pollution. Poisson regression. 


\section{INTRODUCCIÓN}

La ciudad de Zaragoza presenta, de forma habitual, niveles bajos de contaminación atmosférica, por lo que se consideró interesante analizar la posible asociación entre estos niveles de contaminación y la mortalidad por enfermedades respiratorias.

Se seleccionaron las enfermedades respiratorias por haber sido descrita, frecuentemente, la asociación entre mortalidad por las mismas y la contaminación. En nuestra ciudad no hay antecedentes de estudios previos sobre contaminación y mortalidad.

El objetivo de este estudio es evaluar el impacto, a corto plazo, de la contaminación atmosférica sobre la mortalidad respiratoria en la ciudad de Zaragoza, y analizar posibles diferencias existentes en función de la edad y de los tipos de enfermedades respiratorias estudiadas.

\section{MATERIAL Y MÉTODO}

El marco espacial de referencia del estudio ha sido la ciudad de Zaragoza, excluyendo las zonas periurbanas e industriales periféricas. Zaragoza es una ciudad con 572.212 habitantes, según el censo de 1991, que corresponde a la mitad de la población de Aragón.

Se planteó un estudio ecológico en el que se analizó la posible relación entre contaminación atmosférica y mortalidad por enfermedades respiratorias.

\section{Variables analizadas}

Como variable respuesta se ha estudiado el número de defunciones diarias, cuya causa básica de defunción estaba incluida en el grupo de Enfermedades del Aparato Respiratorio (CIE-9 460-519). También se han analizado como variables respuesta el número de defunciones diarias por Neumonía (CIE-9 480-486) y por Enfermedad Pulmo- nar Obstructiva Crónica y Enfermedades Afines, EPOC-EA (CIE-9 490-496).

Se han incluido las defunciones ocurridas durante los años 1991-95, entre las personas residentes de la ciudad de Zaragoza, excluyendo las de las zonas periféricas. Los datos de mortalidad fueron proporcionados por el Instituto Aragonés de Estadística.

Como variables explicativas se han considerado las concentraciones medias diarias de dos indicadores de contaminación atmosférica: humos (partículas en suspensión de diámetro inferior a $10 \mu \mathrm{m}$ ) y dióxido de azufre $\left(\mathrm{SO}_{2}\right)$.

Estos datos se obtuvieron de la Red Manual de Vigilancia del Ayuntamiento, la cual recoge, de manera sistemática, muestras de aire para la determinación de diversos contaminantes, entre los que se encuentran humos y dióxido de azufre. Los métodos de medición utilizados fueron la reflectometría para humos y la espectrofotometría por el método de la Thorina para $\mathrm{SO}_{2}$.

Los valores medios de estos contaminantes se han calculado a partir de la medición realizada en 13 estaciones urbanas (figura 1), habiendo sido excluidas las situadas en áreas industriales.

Los factores de confusión valorados fueron tendencia estacional y a largo plazo; variables climáticas, temperatura y porcentaje de humedad relativa; efectos debidos al día de la semana y festividades; e incidencia diaria de gripe.

Los datos meteorológicos, temperatura y porcentaje de humedad relativa, fueron proporcionados por el Observatorio Meteorológico de Zaragoza.

La incidencia de gripe se ha obtenido a partir del número de casos semanales de gripe notificados al sistema de Enfermedades de Declaración Obligatoria de la Diputación General de Aragón (DGA). 
Figura 1

Lacalización de las estaciones de medición de la contaminación atmosférica. Ciudad de Zaragoza

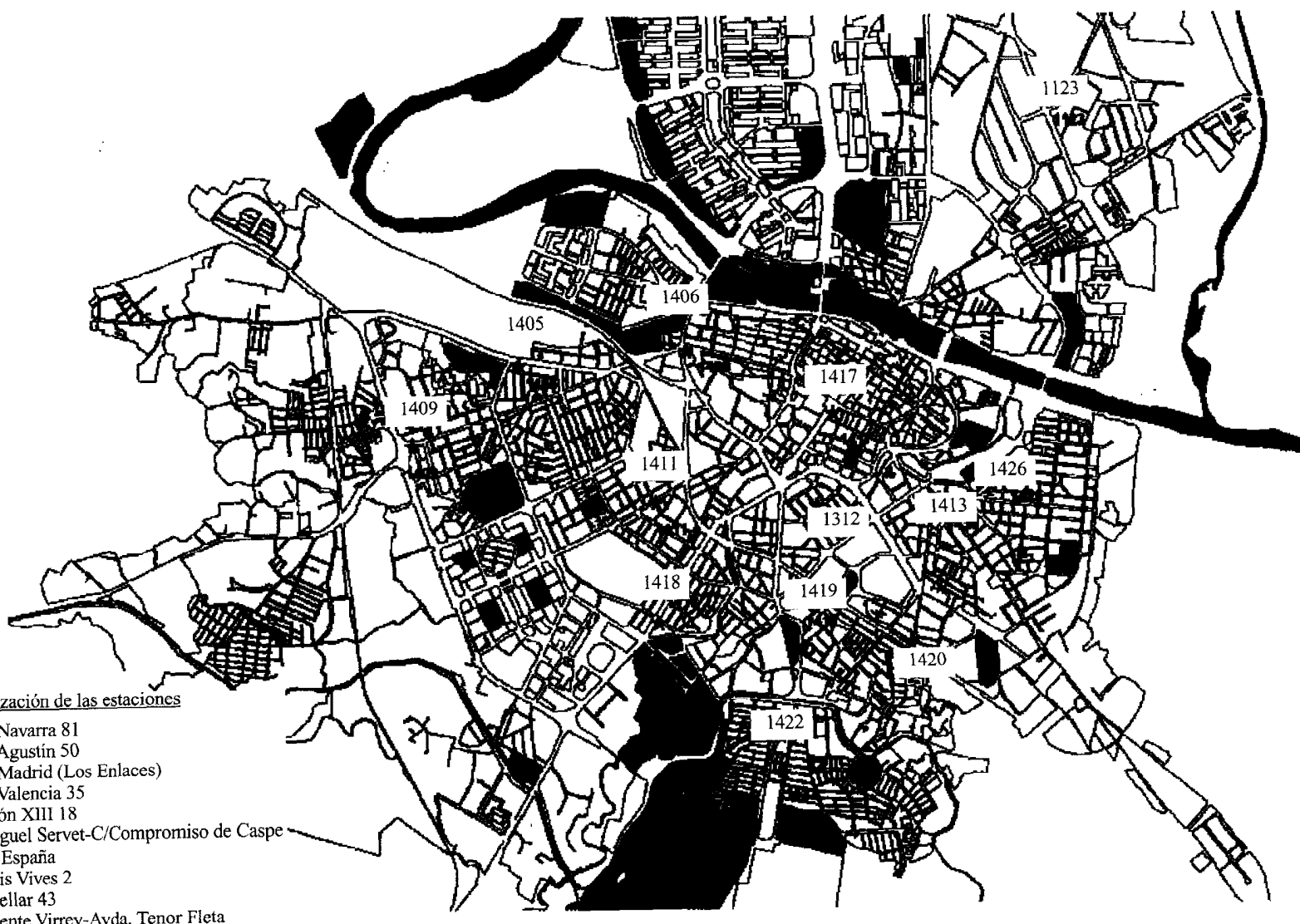

Código Localización de las estaciones

1405 Avda. Navarra 8

$1406 \quad \mathrm{P}^{\mathrm{a}} \mathrm{M}$ Agustin 50

1409 Avda Madrid (Los Enlaces)

1411 Avd Valen

1411 Avda. Valenc

$1413 \mathrm{C} /$ Miguel Servet

1417 Plaza España

1418 C/ Luis Vives

1419 Po Cuellar 43

1420
1422
1123 Avda. América 64

$\begin{array}{lll}\text { N } & 1123 & \text { Avda. Alcalde Caballero } \\ \text { un } & 1426 & \text { C/Monasterio de Siresa ( } \\ & & \end{array}$ 


\section{Método de análisis}

La asociación entre la contaminación atmosférica y la mortalidad diaria se analizó aplicando modelos de regresión de Poisson autorregresiva.

Para identificar posibles diferencias en el efecto de la contaminación según la edad, el análisis se repitió, en el caso de la mortalidad por todas las causas respiratorias, para las defunciones en personas mayores y menores de 70 años.

Los resultados se presentan como riesgo relativo (RR) de mortalidad para un incremento de $10 \mu \mathrm{g} / \mathrm{m}^{3}$ de la concentración de humos y $\mathrm{SO}_{2}$. Se han calculado los intervalos de confianza del Riesgo Relativo al 95\% (IC 95).

\section{RESULTADOS}

De los 22.809 fallecimientos registrados en la ciudad de Zaragoza en el período
1991-95 por todas las causas (excluidas las externas), 2.283 correspondieron a enfermedades del aparato respiratorio. De estas últimas, $1.888(82,7 \%)$ se produjeron en personas de 70 y más años. La media diaria de fallecimientos por enfermedades respiratorias fue para todo el período de $1,25(\mathrm{DE}=1,19)$, con un rango de 0 a 7 . Durante el semestre cálido la mortalidad respiratoria fue de 929 personas, con una media diaria de 1,01 $(\mathrm{DE}=1,04)$, y durante el semestre frío 1354 , con una media de 1,49 (DE=1,27) (tabla 1).

Respecto a los datos de contaminación, el porcentaje de valores válidos osciló entre $91,29 \%$ y $95,35 \%$ para los humos y entre $89,76 \%$ y $93,76 \%$ para $\mathrm{SO}_{2}$, siendo, por tanto, el número de datos no válidos muy bajo. Los valores de los contaminantes tras la imputación de valores perdidos se reflejan en la tabla 2 .

Los datos correspondientes a las variables de control, tanto climatológicas como los casos de gripe, figuran en la tabla 3.

Tabla 1

Mortalidad diaria por enfermedades respiratorias. Zaragoza capital 1991-1995

\begin{tabular}{|lcccc|}
\hline Enfermedades respiratorias & $n$ & Media & Desviación Estándar & Rango \\
\hline Todas las causas & 2.283 & 1,25 & 1,19 & $0-7$ \\
EPOC-EA & 1.011 & 0,55 & 0,77 & $0-7$ \\
Neumonías & 723 & 0,40 & 0,65 & $0-4$ \\
Otras causas & 549 & 0,30 & 0,56 & $0-3$ \\
\hline
\end{tabular}

Fuente: Instituto Aragonés de Estadística. N: Número de defunciones diarias.

EPOC-EA: Enfermedad Pulmonar Obstructiva Crónica y Enfermedades Afines.

Tabla 2

Valores promedio diarios de contaminación. Zaragoza capital 1991-1995

\begin{tabular}{|ccccc|}
\hline & Media & Desviación Estándar & Mediana & Rango \\
\hline Humos $\left(\mu \mathrm{gr} / \mathrm{m}^{3}\right)$ & 46,94 & 21,17 & 44,00 & $5,69-145,38$ \\
Semestre cálido & 42,04 & 17,99 & 39,98 & $5,69-140,85$ \\
Semestre frío & 51,98 & 22,95 & 48,77 & $8,38-145,38$ \\
\hline $\mathrm{SO}_{2}\left(\mu \mathrm{gr} / \mathrm{m}^{3}\right)$ & 21,10 & 15,33 & 17,19 & $0,86-104,58$ \\
Semestre cálido & 12,73 & 8,22 & 11,00 & $0,95-53,54$ \\
Semestre frío & 29,50 & 16,21 & 27,23 & $0,86-104,58$ \\
\hline
\end{tabular}

Fuente: Instituto Municipal de Salud Pública. 
Tabla 3

Datos climatológicos diarios y casos de gripe. Zaragoza capital 1991-1995

\begin{tabular}{|cccc|}
\hline & Media & Desviación Estándar & Rango \\
\hline Temperatura media $\left({ }^{\circ} \mathrm{C}\right)$ & 15,45 & 7,34 & $-1,85-31,80$ \\
Semestre cálido & 21,12 & 5,09 & $7,55-31,80$ \\
Semestre frío & 9,69 & 4,04 & $-1,85-21,75$ \\
\hline Humedad relativa (\%) & 64,46 & 14,92 & $24,00-100,00$ \\
Semestre cálido & 57,73 & 12,38 & $24,00-96,25$ \\
Semestre frío & 71,29 & 14,16 & $30,75-100,00$ \\
\hline Casos de gripe & 169,43 & 202,09 & $5,4-1.375,9$ \\
Semestre cálido & 58,78 & 46,92 & $5,4-237,1$ \\
Semestre frío & 281,79 & 234,60 & $45,6-1.375,9$ \\
\hline
\end{tabular}

Fuente: Observatorio Meteorológico de Zaragoza y Dirección General de Salud Pública-DGA

\section{Efectos asociados a las partículas en suspensión (humos)}

Se encontró una asociación significativa entre mortalidad respiratoria diaria y concentración de humos del día anterior $\mathrm{RR}=1,028\left(\mathrm{IC}_{95 \%}\right.$ 1,006-1,051). En el análisis estratificado por semestres, la asociación se mantuvo para los dos niveles de estratificación, si bien el efecto fue mayor en el semestre cálido, $\mathrm{RR}=1,046\left(\mathrm{IC}_{95 \%}\right.$ 1,005-1,089). En mayores de 70 años esta relación se mantuvo para el semestre cálido, estando al límite de la significación cuando se consideró todo el período de cstudio. En menores de 70 años, se encontraron asociaciones negativas de la concentración de humos dos días antes y la mortalidad respiratoria diaria para todo el periodo. Esta relación fue algo más consistente en el semestre cálido y no se encontraron asociaciones en el semestre frío (tabla 4 y figura 2).

Tabla 4

Influencia de la contaminación por humos sobre la mortalidad respiratoria. Zaragoza capital 1991-1995

\begin{tabular}{|c|c|c|c|c|c|c|c|c|c|}
\hline & \multicolumn{3}{|c|}{ Todo el periodo } & \multicolumn{3}{|c|}{ Semestre cálido } & \multicolumn{3}{|c|}{ Semestre frio } \\
\hline & Ret & $R R$ & IC 95 & Ret. & $R R$ & IC 95 & Ret & $R R$ & IC 95 \\
\hline \multicolumn{10}{|l|}{ Por causas } \\
\hline Todas las causas & 1 & $1,028 * *$ & $1,006-1,051$ & 1 & $1,046^{* *}$ & $1,005-1,089$ & 1 & $1,024^{*}$ & $0,997-1,050$ \\
\hline EPOC-EA & 5 & $1,038 * *$ & $1,002-1,075$ & 5 & $1,068 * *$ & $1,004-1,137$ & 5 & 1,029 & $0,989-1,070$ \\
\hline Neumonias & 4 & 0,979 & $0,940-1,020$ & 4 & 1,054 & $0,981-1,133$ & 4 & $0,952 * *$ & $0,907-0,999$ \\
\hline \multicolumn{10}{|l|}{ Por edades } \\
\hline Todas las edades & 1 & $1,028 * *$ & $1,006-1,051$ & 1 & $1,046 * *$ & $1,005 \sim 1,089$ & 1 & $1,024 *$ & $0,997-1,050$ \\
\hline 70 años y más & 1 & $1,025^{*}$ & $0,999-1,051$ & 1 & $1,048^{* *}$ & $1,005-1,094$ & 1 & 1,016 & $0,987-1,046$ \\
\hline$<70$ años & 2 & $0,940^{*}$ & $0,883-1,000$ & 2 & $0,859 * *$ & $0,767-0,962$ & 5 & 1,040 & $0,974-1,109$ \\
\hline
\end{tabular}

EPOC-EA: Enfermedad Pulmonar Obstructiva Crónica y Enfermedades Afines.

Ret: Retardo en dias.

RR: Riesgo Relativo de mortalidad por aumento de la concentración de humos en $10 \mu \mathrm{gr} / \mathrm{m}^{3}$.

IC 95: Intervalo de confianza al $95 \%$.

** $\mathrm{p}<0,05 * \mathrm{pp}<0,10$.

Nota: Se incluyen los retardos con mayor nivel de significación en cada periodo 
Figura 2

Riesgo Relativos de mortalidad diaria por causas respiratorias asociados a cambios en la concentración de humos. Zaragoza, 1991-1995

\section{SEGÚN CAUSAS}

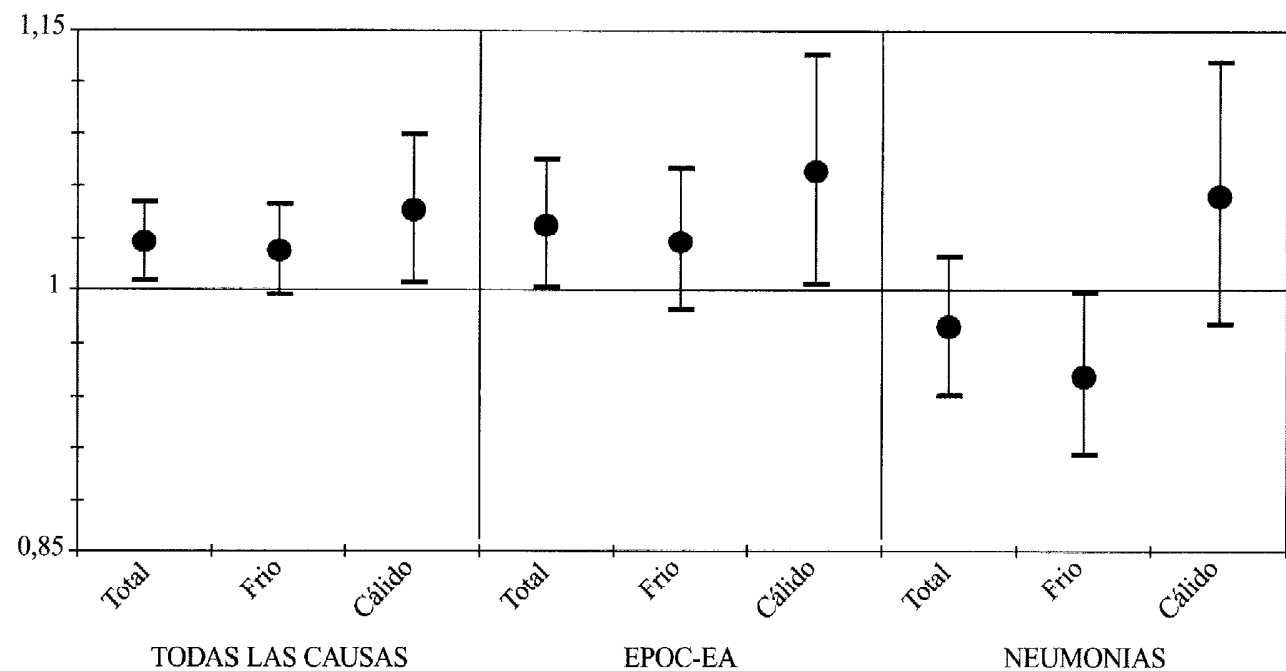

\section{SEGÚN EDADES}

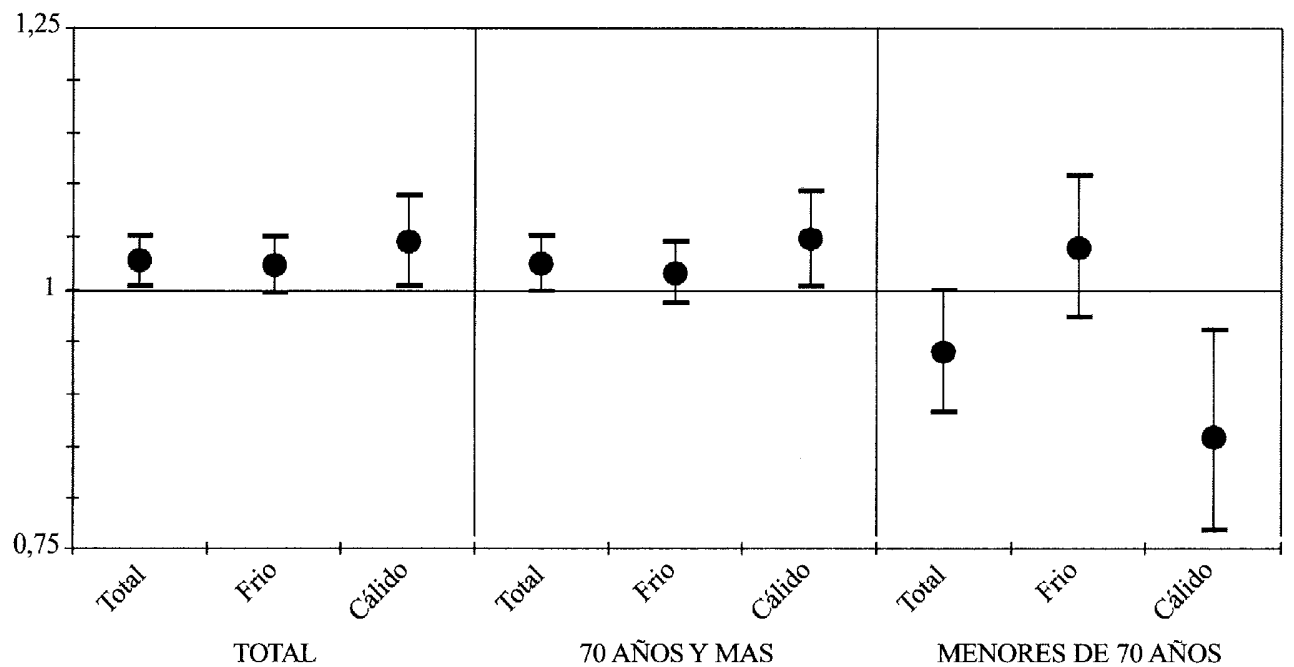

EPOC-EA: Enfermedad Pulmonar Obstructiva Crónica y Enfermedades Afines. Riesgo Relativo de mortalidad por aumento de la concentraciön de humos en $10 \mu \mathrm{gr} / \mathrm{m}^{3}$. Intervalos de confianza calculados al 95\%. Nota: Se incluyen los retardos con mayor nivel de significación en cada período. 
Dentro de la mortalidad respiratoria, los resultados para EPOC-EA y neumonía se presentan en la tabla 4 y en la figura 2 . Destacan las asociaciones encontradas entre la mortalidad por EPOC y la contaminación por humos cinco días antes, con $\mathrm{RR}=1,038$ $\left(\mathrm{IC}_{95 \%}\right.$ 1,002-1,075) para todo el período $\mathrm{y}$ $\mathrm{RR}=1,068\left(\mathrm{IC}_{95 \%}\right.$ 1,004-1,137) para el período cálido. En el caso de la mortalidad por neumonía, los efectos detectados para el período cálido y frío fueron de signo opuesto, destacando que en el período frío, la asociación encontrada fue negativa, $\mathrm{RR}=0,952$ ( $\mathrm{IC}_{95 \%}$ 0,907-0,999).

\section{Efectos asociados al $\mathrm{SO}_{2}$}

Cuando se analizó todo el período, no se detectaron asociaciones entre $\mathrm{SO}_{2}$ y mortalidad respiratoria por todas las causas. En el período cálido se detectó una asociación positiva significativa entre la concentración de $\mathrm{SO}_{2} 4$ días antes y la mortalidad respiratoria, para todas las edades $\left(\mathrm{RR}=1,093\right.$; $\mathrm{IC}_{95 \%}$ : 1,006-1,187). En menores de 70 años se detectó la misma asociación $(\mathrm{RR}=1,240$; $\left.\mathrm{IC}_{95 \%}: 1,028-1,496\right)$, mientras que los resul- tados para los mayores de 70 años no fueron concluyentes (ver tabla 5 y figura 3 ).

Se encontró asociación estadísticamente significativa positiva entre la contaminación por $\mathrm{SO}_{2}$ y la mortalidad por EPOC-EA en el semestre cálido $\left(\mathrm{RR}=1,135 ; \mathrm{IC}_{95 \%}\right.$ : 1,0011,287). En el caso de la mortalidad por neumonía, la asociación con la contaminación por $\mathrm{SO}_{2} \mathrm{sc}$ mantuvo en el límite de significación tanto en el semestre frío $(\mathrm{RR}=1,063$; $\left.\mathrm{IC}_{95 \%}: 0,995-1,135\right)$, como para todo el período $\left(\mathrm{RR}=1,059 ; \mathrm{IC}_{95 \%}: 0,997-1,125\right)$ (ver tabla 5 y figura 3 ).

\section{CONCLUSIONES}

Los resultados del trabajo confirman los hallazgos de otros estudios, que demuestran el efecto perjudicial sobre la salud que, a corto plazo, pueden tener niveles de contaminación que suelen considerarse segu$\operatorname{ros}^{1-5}$.

Se encontró una asociación significativa entre humos y $\mathrm{SO}_{2}$ y la mortalidad por enfermedades respiratorias. Esta asociación se detectó con niveles de contaminación muy

Tabla 5

Influencia de la contaminación por dióxido de azufre $\left(\mathrm{SO}_{2}\right)$ sobre la mortalidad respiratoria. Zaragoza capital 1991-1995

\begin{tabular}{|c|c|c|c|c|c|c|c|c|c|}
\hline & \multicolumn{3}{|c|}{ Todo el periodo } & \multicolumn{3}{|c|}{ Semestre cálido } & \multicolumn{3}{|c|}{ Semestre frio } \\
\hline & Ret & $R R$ & IC 95 & Ret. & $R R$ & IC 95 & Ret & $R R$ & IC 95 \\
\hline \multicolumn{10}{|l|}{ Por causas } \\
\hline Todas las causas & 3 & 0,983 & $0,948-1,019$ & 4 & $1,093 * *$ & $1,006-1,187$ & 3 & 0,982 & $0,945-1,021$ \\
\hline EPOC-EA & 1 & 1,038 & $0,980-1,099$ & 4 & $1,135 * *$ & $1,001-1,287$ & 4 & 1,021 & $0,960-1,086$ \\
\hline Neumonias & 1 & $1,059^{*}$ & $0,997-1,125$ & 1 & 1,039 & $0,895-1,206$ & 1 & $1,063^{*}$ & $0,995-1,135$ \\
\hline \multicolumn{10}{|l|}{ Por edades } \\
\hline Todas las edades & 3 & 0,983 & $0.948-1,019$ & 4 & $1,093 * *$ & $1,006-1,187$ & 3 & 0,982 & $0,945-1,021$ \\
\hline 70 años y más & 2 & 1,030 & $0,990-1,072$ & 4 & 1,069 & $0,974-1,173$ & 2 & 1,035 & $0,992-1,080$ \\
\hline$<70$ años & 4 & 1,059 & $0,963-1,165$ & 4 & $1,240 * *$ & $1,028-1,496$ & 3 & 0,956 & $0,861-1,061$ \\
\hline
\end{tabular}

EPOC-EA: Enfermedad Pulmonar Obstructiva Crónica y Enfermedades Afines.

Ret: Retardo en días.

RR: Riesgo Relativo de mortalidad por aumento de la concentración de $\mathrm{SO}_{2}$ en $10 \mu \mathrm{gr} / \mathrm{m}^{3}$.

IC 95: Intervalo de confianza al $95 \%$.

${ }^{* *} \mathrm{p}<0,05^{*} \mathrm{pp}<0,10$.

Nota: Se incluyen los retardos con mayor nivel de significación en cada período. 
Figura 3

Riesgos relativos de mortalidad diaria por causas respiratorias asociados a cambios en la concentración de $\mathrm{SO}_{2}$. Zaragoza, 1991-1995.

\section{SEGÚN CAUSAS}

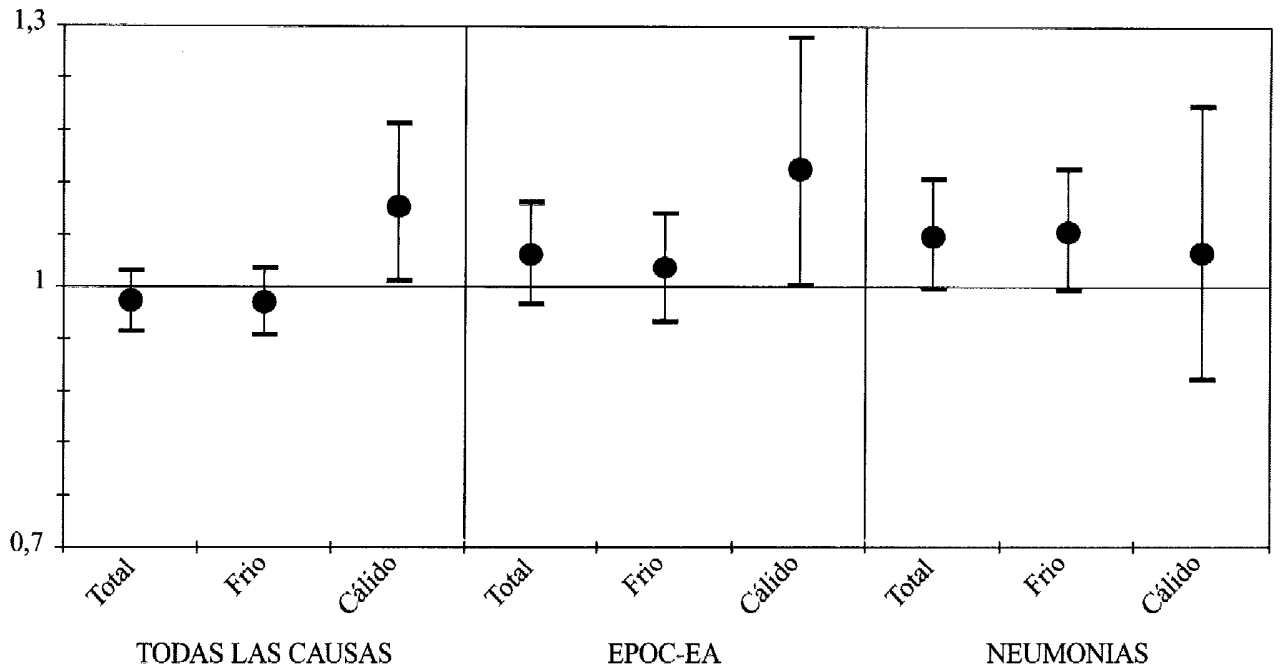

\section{SEGÚN EDADES}

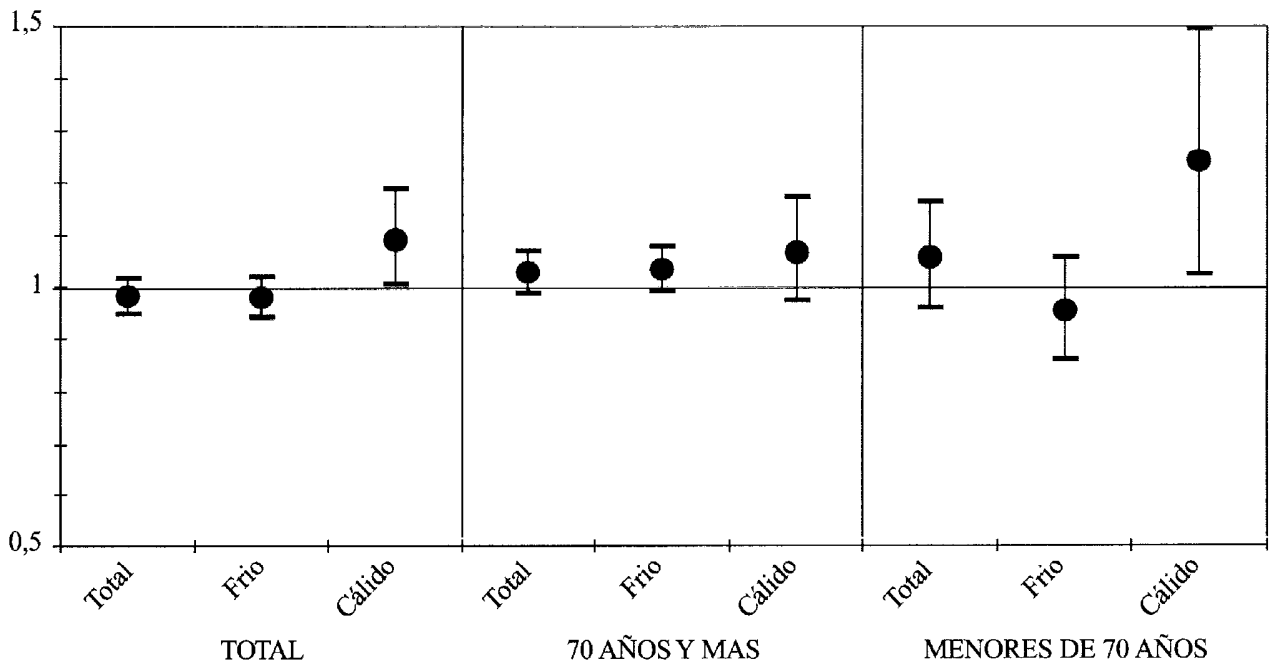

EPOC-EA: Enfermedad Pulmonar Obstructiva Crónica y Enfermedades Afines. Riesgo Relativo de mortalidad por aumento de la concentración de $\mathrm{SO}_{2}$ en 10 $\mu \mathrm{gr} / \mathrm{m}^{3}$. Intervalos de confianza calculados al 95\%. Nota: Se incluyen los retardos con mayor nivel de significación en cada período. 
bajos, característicos de la ciudad de Zaragoza. El Real Decreto 1613/1985 de normas de calidad de $\mathrm{SO}_{2}$ y partículas en suspensión, indica que los valores guía para estos contaminantes, en un período de 24 horas, es de $100-150 \mu \mathrm{gr} / \mathrm{m}^{3} \mathrm{~N}$ (valor medio diario) y para un período anual (media aritmética de los valores medios diarios del año) de 40 a $60 \mu \mathrm{gr} / \mathrm{m}^{3}$, valores superiores a los encontrados en nuestro estudio.

Se observó un incremento del $2,8 \%$ en la mortalidad respiratoria global por cada aumento de $10 \mu \mathrm{g} / \mathrm{m}^{3}$ en la concentración media diaria de humos. En el caso del $\mathrm{SO}_{2}$ no se pudo demostrar esta asociación cuando se analizaron conjuntamente los dos períodos.

La mortalidad por todas las causas respiratorias en Zaragoza se incrementó de forma significativa, tanto para humos como para $\mathrm{SO}_{2}$ en el período cálido, mientras que en el período frío no se pudo demostrar de forma clara el efecto de los contaminantes. Esta diferencia de efecto en el período cálido fue más importante en el caso del $\mathrm{SO}_{2}$.

Las diferencias encontradas podrían deberse a una menor exposición de las personas a los contaminantes exteriores en invierno y a que la mortalidad por causas infecciosas, o las relacionadas con baja temperatura, tiene menor peso en verano. El mayor efecto del $\mathrm{SO}_{2}$ en verano, tambićn pucde estar relacionado con el efecto de otros contaminantes no analizados en el estudio. Por ejemplo ha sido descrita la influencia de los niveles de ozono sobre la mortalidad ${ }^{6-9}$, que en algunos estudios ha sido más elevada en períodos cálidos ${ }^{10}$.

Se han detectado efectos discordantes de la contaminación por humos según los grupos de edad analizados. En el grupo de mayor edad la asociación ha sido positiva mientras que en los menores de 70 años la asociación fue negativa. En el caso de la contaminación por $\mathrm{SO}_{2}$, no se encontraron diferencias relevantes en función de la edad.
En el análisis según tipos de enfermedades respiratorias, nuestros datos muestran una clara asociación entre incrementos en la concentración de humos y mortalidad por EPOC-EA, mientras que en el caso de la mortalidad por neumonías la asociación es más evidente con el $\mathrm{SO}_{2}$.

En resumen, el estudio indica que niveles bajos de contaminación pueden producir efectos significativos sobre la mortalidad respiratoria, en especial en períodos cálidos y en personas mayores. Lo anterior evidencia el riesgo implícito asumido con los actuales estándares y normas técnicas y cuestiona la seguridad de los niveles generalmente aceptados en el momento presente.

\section{BIBLIOGRAFÍA}

1. Leuenberger $P$, Kunzli N, Ackermann Liebrich U, Schindler C, Bolognini G, Bongard JP, Brandli O, Defila C, Domenighetti G, Karrer W, Keller R, Medici T, Monn C, Perruchoud AP, Schoni M, Tschopp JM, Villiger B, Wuthrich B, Zellweger JP. Swiss Study on Air Pollution and Lung Diseases in Adults (SAPALDIA). Schweiz Med Wochenschr 1998; 128: 150-61.

2. Sunyer J, Castellsague J, Saez M, Tobías A, Antó JM. Air pollution and mortality in Barcelona. $\mathrm{J}$ Epidemiol Community Health 1996; 50 Supl 1: 76-80.

3. Vigotti MA, Rossi G, Bisanti L, Zanobetti A, Schwartz J. Short term effects of urban air pollution on respiratory health in Milan, Italy, 198089. J Epidemiol Community Health 1996; 50 Supl 1: 71-5.

4. Thurston GD. A critical review of PM10-mortality time-series studies. J Expo Anal Environ Epidemiol 1996; 6: 3-21.

5. Salinas M and Vega J. The effect of outdoor air pollution on mortality risk: an ecological study from Santiago, Chile. World Health Stat Q 1995; 48: $118-25$

6. Spix C, Anderson HR, Schwartz J, Vigotti MA. LeTertre A, Vonk JM, Touloumi G, Balducci F, Piekarski T, Bacharova L, Tobias A, Ponka A, Katsouyanni K. Short-term effects of air pollution on hospital admissions of respiratory diseases in Europe: a quantitative summary of APHEA study results. Air Pollution and Health: a European Approach. Arch Environ Health 1998; 53: 54-64. 
7. Simpson RW, Williams G, Petroeschevsky A, Morgan G, Rutherford S. Associations between outdoor air pollution and daily mortality in Brisbane, Australia. Arch Environ Health 1997; 52: 442-54.

8. Hoek G, Schwartz JD, Groot B, Eilers P. Effects of ambient particulate matter and ozone on daily mortality in Rotterdam, The Netherlands. Arch Environ Health 1997; 52: 455-63.
9. Kelsall JE, Samet JM, Zeger SL, Xu J. Air pollution and mortality in Philadelphia, 1974-1988. Am J Epidemiol 1997; 146: 750-62.

10. Sartor F, Snacken R, Demuth C and Walckiers D. Temperature, ambient ozone levels, and mortality during summer 1994, in Belgium. Environ Res 1995; 70: 105-13. 